\title{
Inverted Leidenfrost-like Effect during Condensation
}

Ramchandra Narhe, ${ }^{\dagger \dagger}$ Sushant Anand, ${ }^{\S}$ Konrad Rykaczewski, ${ }^{\dagger}$ Marie-Gabrielle Medici, ${ }^{\dagger, \perp}$ Wenceslao González-Viñas, ${ }^{\ddagger}$ Kripa K. Varanasi, ${ }^{\S}$ and Daniel Beysens ${ }^{*}, \dagger, \#$

†Physique et Mécanique des Milieux Hétérogènes, Unité Mixte de Recherches (UMR) 7636, École Supérieure de Physique et Chimie Industrielles ParisTech, Université Pierre et Marie Curie, Université Paris-Diderot, Centre National de la Recherche Scientifique (CNRS), 10 rue Vauquelin, 75231 Paris, France

${ }^{\ddagger}$ Department of Physics and Applied Mathematics, University of Navarra, 31008 Pamplona, Spain

${ }^{\S}$ Department of Mechanical Engineering, Massachusetts Institute of Technology, Cambridge, Massachusetts 02139, United States

"School for Engineering of Matter, Transport and Energy, Arizona State University, Tempe, Arizona 85281, United States

${ }^{\perp}$ Université Nice Sophia Antipolis, CNRS, Laboratoire de Physique de la Mateière Condensée-UMR 7336, Parc Valrose, 06100 Nice, France

${ }^{\#}$ Service des Basses Températures, CEA-Grenoble and Université Joseph Fourier, 38041 Grenoble, France

\section{Supporting Information}

ABSTRACT: Water droplets condensing on solidified phase change materials such as benzene and cyclohexane near their melting point show in-plane jumping and continuous "crawling" motion. The jumping drop motion has been tentatively explained as an outcome of melting and refreezing of the materials surface beneath the droplets and can be thus considered as an inverted Leidenfrost-like effect (in the classical case vapor is generated from a droplet on a hot substrate). We present here a detailed investigation of jumping movements using high-speed imaging and static crosssectional cryogenic focused ion beam scanning electron microscope imaging. Our results show that drop motion is induced by a

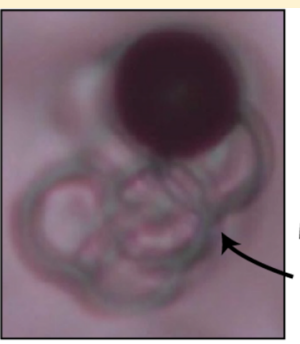

Hopping

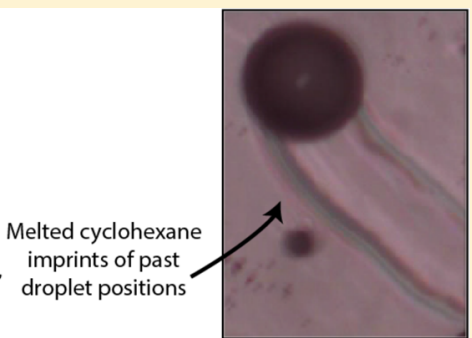

Crawling thermocapillary (Marangoni) effect. The in-plane jumping motion can be delineated to occur in two stages. The first stage occurs on a millisecond time scale and comprises melting the substrate due to drop condensation. This results in droplet depinning, partial spreading, and thermocapillary movement until freezing of the cyclohexane film. The second stage occurs on a second time scale and comprises relaxation motion of the drop contact line (change in drop contact radius and contact angle) after substrate freezing. When the cyclohexane film cannot freeze, the droplet continuously glides on the surface, resulting in the crawling motion.

\section{INTRODUCTION}

Manipulating liquid droplets on surfaces is important for numerous areas such as energy generation, microfluidics, electronics, icephobicity, antifogging, etc. Droplet manipulation can be obtained using passive techniques such as wettability gradients or "designed" surface textures, ${ }^{1-4}$ as well as using active techniques where droplets may respond to external stimuli such as vibrations, ${ }^{5-7}$ light, ${ }^{8,9}$ electric fields, ${ }^{10}$ acoustics, ${ }^{11}$ magnetic fields, ${ }^{12,13}$ or cyclical mechanical deformation of the substrate. ${ }^{14}$ In addition to the abovementioned methods, droplet manipulation in many heat transfer applications can also be obtained passively using in situ phase change processes as exemplified by the Leidenfrost effect $^{15,16}$ (Figure 1a), self-propelled motion during evaporation, ${ }^{17,18}$ and water droplet motion during condensation on phase change materials (PCMs). ${ }^{19,20}$ The latter phenomenon, first reported by Steyer et al., ${ }^{19}$ was observed during condensation of water on solid cyclohexane kept at a temperature $T_{\mathrm{c}}$ below its melting temperature $\left(T_{\mathrm{m}}=6.52\right.$ $\left.{ }^{\circ} \mathrm{C}\right) .{ }^{21}$ The drop motion has been explained as an outcome of melting and refreezing of the cyclohexane surface beneath the droplets. This mechanism is similar to the inverted Leidenfrost effect $^{22-26}$ where the stored heat in an object or droplet can cause vapor generation from the surrounding medium, which could be either a liquid ${ }^{22-24}$ or a solid ${ }^{25,26}$ (Figure 1b). However, here the heat released during condensation-assisted droplet growth results in a phase change from solid to liquid of the substrate as opposed to liquid to vapor in the conventional Leidenfrost effect, or solid to vapor in some cases of the inverted Leidenfrost effect. ${ }^{25,26}$ In the classical Leidenfrost problem, the droplet is in a nonwetting state with no distinct contact line. ${ }^{15,27}$ On the other hand, condensing droplets on a PCM such as cyclohexane (Figure 1c) are in contact with the underlying substrate (which alters between the liquid and solid

Received: December 16, 2014

Revised: March 14, 2015

Published: March 25, 2015 


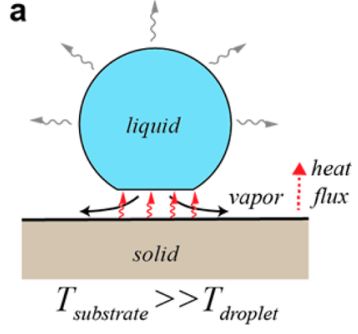

d

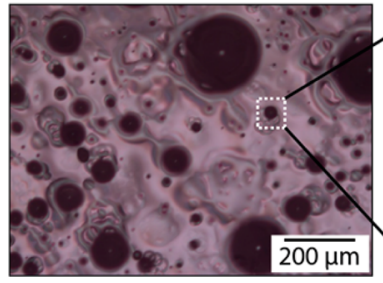

b

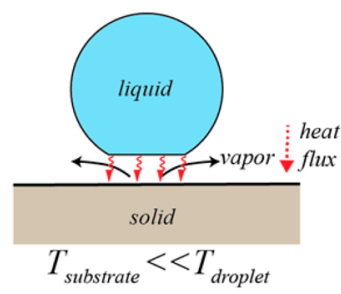

e
C

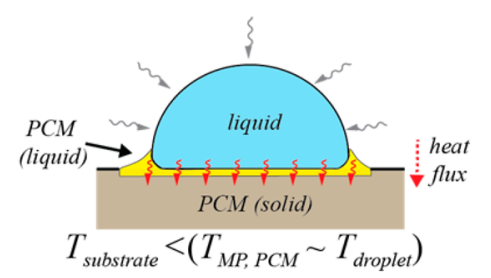

Figure 1. (a) Schematic showing the classical Leidenfrost phenomenon. (b) Schematic of the inverse Leidenfrost phenomenon on solids that undergo sublimation upon heat absorption. ${ }^{25,26}$ (c) Schematic of inverse Leidenfrost-like behavior during condensation on substrates that undergo melting upon heat absorption. (d, e) Optical observation of water condensation on cyclohexane and forms of motion exhibited by condensing droplets. (d) In-plane jumping motion. The inset highlights the traces left by the drops at each jump. Experimental conditions: $T_{\mathrm{a}}=21{ }^{\circ} \mathrm{C}, \mathrm{RH}=$ $38 \%, T_{\mathrm{c}}=1{ }^{\circ} \mathrm{C}$, natural convection. (e) Crawling motion. The inset highlights the trace left by the drop. In the right part, two drops undergoing jumping collide but do not immediately coalesce due to the liquid cyclohexane film between the drops. The semicoalesced drops produce a sustained motion lasting several droplet diameters. Experimental conditions: $T_{\mathrm{a}}=21^{\circ} \mathrm{C}, \mathrm{RH}=30 \%, T_{\mathrm{c}}=2{ }^{\circ} \mathrm{C}$, natural convection.

state). Despite this difference, the condensing droplets on cyclohexane move freely on the underlying liquid layer as the hysteresis on the liquid surface is negligible, ${ }^{28}$ similar to the hysteresis free motion of Leidenfrost droplets on the vapor layer. Note that the motion of condensed droplets on PCMs near their melting point is representative of an isothermal microsteam engine. Usual steam engines are adiabatic; however, this condition cannot be maintained on the microscale where the typical heat diffusion times become too small.

The focus of the present work is elucidating the detailed mechanisms governing droplet motion during condensation on a PCM (near its melting point). The initial observations by Steyer et al. ${ }^{19}$ showed that, for water drops condensing on solid cyclohexane near its melting temperature, the droplet growth was accompanied by in-plane rotation, translation, and seemingly "hopping" or stick/slip-like movements (Figure 1d). In addition to these stick/slip movements, another droplet propulsion behavior involving continuous motion of droplets (referred to as drop crawling) has also been observed (Figure 1e). ${ }^{19}$ This motion can be observed concurrently with the hopping motion of other drops. However, as time goes on, hopping motion is recovered, making the stick/slip mechanism the most common.

The first interpretation of the in-plane jumping motion was based on the formation of a cyclohexane ridge due to elastic deformation of the substrate. The relaxation of the substrate strain during localized solid melting at the drop perimeter was said to induce the motion (Figure 1d). The droplet motion was facilitated by the presence of a melted film under the drop, and the motion stopped as the film froze. After a time $t_{\mathrm{m}}$, a melting layer was formed again due to condensation, and subsequently, the droplet motion was reinitiated. The melting time $t_{\mathrm{m}}$ is the time required for heating of cyclohexane to its melting temperature $T_{\mathrm{m}}$ by the release of the latent heat of water condensation. This time was found to scale with the drop contact radius $R$ as

$$
t_{\mathrm{m}}=\alpha R
$$

For the experimental conditions ${ }^{19} T_{\mathrm{c}}=3.4{ }^{\circ} \mathrm{C}$, air temperature $T_{\mathrm{a}}=24.2{ }^{\circ} \mathrm{C}, \mathrm{RH}$ (relative humidity) $=100 \%$, and air flux $F=0.17 \mathrm{~L} / \mathrm{min}$, it was found that $\alpha \approx 6 \times 10^{4} \mathrm{~s}$. $\mathrm{m}^{-1}$. It was also observed that, after moving, a drop leaves a circular print. However, it was unclear whether the circular print was a liquid or solid ridge (upward deformation) or trench (downward deformation).

The interpretation of elastic deformation of the substrate was revisited by Katz. ${ }^{29} \mathrm{He}$ calculated the released elastic energy and found that it was significantly smaller than the change in capillary energy corresponding to the difference in drop contact angle on liquid and solid cyclohexane. Katz's explanation was that the drop rolls on a film of melted cyclohexane and stops after its freezing. Substrate melting should cause formation of a residual trench. Experiments were performed by Narhe et al. ${ }^{20}$ on water condensation on benzene below its melting point $\left(T_{\mathrm{m}}\right.$ $=5.5{ }^{\circ} \mathrm{C}$ ). They confirmed the Steyer et al. observations concerning the condensation-induced jumping behavior. For benzene the value $\alpha \approx 8.5 \times 10^{4} \mathrm{~s} \cdot \mathrm{m}^{-1}$ was found, which is close to the above value measured for cyclohexane. Narhe et $\mathrm{al}^{20}$ provided a new interpretation of the mechanisms involving substrate melting at the drop perimeter in an annulus of constant cross-section. A proportionality relation between the jumping length $s_{\mathrm{j}}$ and the drop contact radius was obtained:

$$
s_{\mathrm{j}}=\beta R
$$

with $\beta \approx 1.5$. A sudden increase of drop radius during movement was also detected. After the drop stops, the radius slowly decreases to its initial value $R_{0}$. Only a minor increase in the radius past $R_{0}$ was observed, due to condensation. The drop contact radius was observed to follow an exponential decay to a value $R_{\infty}$ with typical time $t_{\mathrm{c}}$ :

$$
R=R_{\infty}+\left(R_{0}-R_{\infty}\right) \exp \left(-t / t_{c}\right)
$$

Although previous studies have shed important insights on water condensation on phase change materials occurring on large time scales, the various mechanistic processes that occur 
during this phenomenon cannot be fully understood without further information about the short time scale process dynamics, contact angle measurements, and topographical characterization of the droplet imprint (i.e., ridge vs trench). In the present study, we describe new dynamic observations using side- and top-view high-speed imaging and static crosssectional observations acquired using a cryogenic focused ion beam scanning electron microscope (cryo-FIB-SEM) to describe the jumping motion of the droplets. We focus on the behavior of a drop between two coalescence events since droplet coalescence only affects the growth $\operatorname{law}^{20}$ and does not interfere with the in-plane jumping phenomenon. We elucidate the role of condensation-induced melting during various stages of droplet motion. In addition to depinning the drop from the substrate, melting also changes the shape of the drop, gives it a thrust, and induces thermal gradients that provoke drop thermocapillary motion. This behavior is similar to observations on the inverted Leidenfrost effect; ${ }^{22-26}$ however, here the type of droplet motion is related to the thickness of the melted cyclohexane film. In particular, a thin melt film that can freeze rapidly induces the intermittent drop motion through refreezing and remelting. When the film is thick, freezing does not occur and a steady crawling motion occurs. We focus on the jumping motion, because it is the most common behavior (the crawling motion, although a variant of the same phenomenon, deserves another specific study). The presented observations and supporting analysis provide for the first time a comprehensive description of the in-plane jumping motions.

\section{EXPERIMENTAL METHODS}

2.1. Optical Observation. The condensation process was observed on horizontal substrates, looking from above using an optical microscope or from the side by using a macrolens. Condensation was performed either in a chamber where air was sent at $100 \%$ relative humidity and at room temperature or by directly cooling the samples in the room atmosphere. In all cases the air temperature and humidity were stable (air temperature within $0.5{ }^{\circ} \mathrm{C}$ and relative humidity within $3 \%$ ).

2.1.1. Top-Down Light Microscope Observation. The experimental setup is identical to the one described by Steyer et al. ${ }^{19}$ and Narhe et al. ${ }^{20}$ Briefly, the condensation chamber consists of a Peltier-element thermostat enclosed in a Plexiglas box.

In the first set of experiments, a small piece of cleaned silicon wafer of size $1 \times 1 \mathrm{~cm}^{2}$ is fixed on a thick electrolytic copper plate of diameter $L=100 \mathrm{~mm}$ by means of a thin film of water to reduce the contact resistance between the substrate and the cold plate. In the beginning, a small drop $(\sim 20 \mu \mathrm{L})$ of cyclohexane (purity $99.99 \%)$ is deposited on the wafer, where it forms a film of $1-1.5 \mathrm{~mm}$ thickness, which slowly evaporates. It is then cooled from room temperature (typically $T_{\mathrm{a}} \approx 23{ }^{\circ} \mathrm{C}$ ) to a temperature (typically $T_{\mathrm{c}}=3{ }^{\circ} \mathrm{C}$ ) well below the melting temperature of cyclohexane $\left(T_{\mathrm{m}}=6.7^{\circ} \mathrm{C}\right)$. The substrate temperature is measured by a K-type thermocouple taped on the copper surface. Air flow saturated with water vapor at room temperature with flux $F \approx 0.2 \mathrm{~L} / \mathrm{min}$ is sent into the chamber. The jumping dynamics of the drops is recorded either with a chargecoupled device (CCD) camera (COHU, 4910 series, $50 \mathrm{fps}$ ) or a highresolution color camera (PixeLink PL B776F) operating at $1 \mathrm{fps}$. A high-speed camera (Phantom V9.1) was also used. The cameras are attached to an optical microscope (Leica, DMRXE). The high-speed camera is able to capture $10^{3} \mathrm{fps}$ at $1632 \times 1200$ pixels $^{2}$ and up to $10^{5}$ fps at $128 \times 8$ pixels $^{2}$, at 14 bit resolution. However, use of high frame rates in condensation experiments is limited by the need for intense illumination. In particular, when intense light was used, the substrate melted immediately and no jumping phenomena happened. The highest frame rate that we achieved without melting cyclohexane was $4000 \mathrm{fps}$, with an exposure time of $208 \mu$ s and a frame interval of 250 $\mu$ s. This time is not short enough to resolve the details of substrate melting. However, it is much smaller than the time interval in the previous studies $(20 \mathrm{~ms}) .{ }^{19}$

In the second set of experiments, a cyclohexane film is formed at the bottom of hollow blocks of either polyethylene $(29.1 \mathrm{~mm}$ diameter, $6.5 \mathrm{~mm}$ height, $4.5 \mathrm{~mm}$ depth, $1.4 \mathrm{~mm}$ thickness) or copper $(20.0 \mathrm{~mm}$ diameter, $4.8 \mathrm{~mm}$ depth, $10.0 \mathrm{~mm}$ height, $1.5 \mathrm{~mm}$ thickness). The blocks are stuck with a layer of water on the same Peltier-cooled copper plate as used in the above condensation chamber. Temperature is measured on the electrolytic copper block. The images are analyzed by Image software. ${ }^{30}$

2.1.2. Side-View Optical Observation. For observing droplets from the side (droplet diameter 5-100 $\mu \mathrm{m}$ ), the growth process is observed with a high-resolution CCD color camera (PixeLink, PL B776F) connected to a computer. This camera is equipped with a $18 \mathrm{~mm} / 200$ $\mathrm{mm}$ zoom lens. Cyclohexane (1-1.5 mm thickness) is put in the same hollow piece of polyethylene as described just above in section 2.1.1 and cooled the same way. A $2 \mu \mathrm{L}$ drop of water is deposited with a syringe. Note that tiny droplets condense on the rim around the large drop (see Figure 2a). The substrate temperature is measured by a Ktype thermocouple placed on the surface of the copper block.

2.2. Cryo-FIB-SEM Imaging. For direct imaging of the dropcyclohexane interfaces, we used a modification of the cryo-FIB-SEM method, which has been recently used to image water drops ${ }^{31,32}$ on superhydrophobic and lubricant-impregnated surfaces. In particular, custom copper stubs with about $2 \mathrm{~mm}$ deep and wide holes were fabricated to contain the cyclohexane. This copper "cup" was mounted to the cryo shuttle transfer device and filled with cyclohexane. As in our prior work, to trigger condensation on the cyclohexane surface, the entire assembly was cooled using an ice-bath-cooled Peltier element. The temperature of the copper cup was monitored using a thermocouple mounted in a hole mounted on the side on the cup. The condensation process was monitored using an optical microscope. The sample temperature was adjusted until the droplet motion was observed. Typical experimental conditions are a temperature of about $20{ }^{\circ} \mathrm{C}$ and a relative humidity of $40-45 \%$. The sample was cooled to $3-4{ }^{\circ} \mathrm{C}$.

Subsequently, the sample was plunge-frozen in liquid nitrogen slush. The freezing rate for the process is about $2 \times 10^{4} \mathrm{~K} \cdot \mathrm{s}^{-1}$, resulting in $\mu \mathrm{s}$ freezing times for the microdroplets on the surface. The same transfer, grounding, FIB milling, and SEM imaging procedures as in our previous work were utilized. ${ }^{31-33}$ Preservation of two liquid phase wetting states (e.g., microdroplets of water on oil) has been well documented, ${ }^{31}$ so the wetting state of a drop on cyclohexane is also unlikely to be affected in our experiments. In this process, deposition of a grounding $\mathrm{Au} / \mathrm{Pd}$ thin film followed by thicker Pt film deposition for the cutting process leads to surface roughness. There is also some possibility of a very small $(<100 \mathrm{~nm})$ nonuniform layer of water nanocrystals frozen onto the surfaces underneath the other two films. This could have formed during the freezing/transport process from residual moisture in air. However, the above-mentioned artifacts do not play any significant role in the imaging of droplets on the substrate.

\section{RESULTS AND DISCUSSION}

3.1. Onset of Droplet Motion. The propulsion of an individual droplet is associated with the intermittent freezing and melting behavior of the cyclohexane substrate in contact with the droplet. The intermittent phase change of the substrate implies that the droplet-substrate interfacial tension also varies intermittently from water-solid to water-liquid. Since the surface energy manifests in the form of a water droplet contact angle on the substrate, the contact angles of water on liquid and solid cyclohexane are expected to differ. If the contact angles differ significantly, the change in contact angle may be expected to play an important role in droplet propulsion. To clarify the role of droplet-substrate interactions in droplet propulsion, we investigate the morphology of a water 

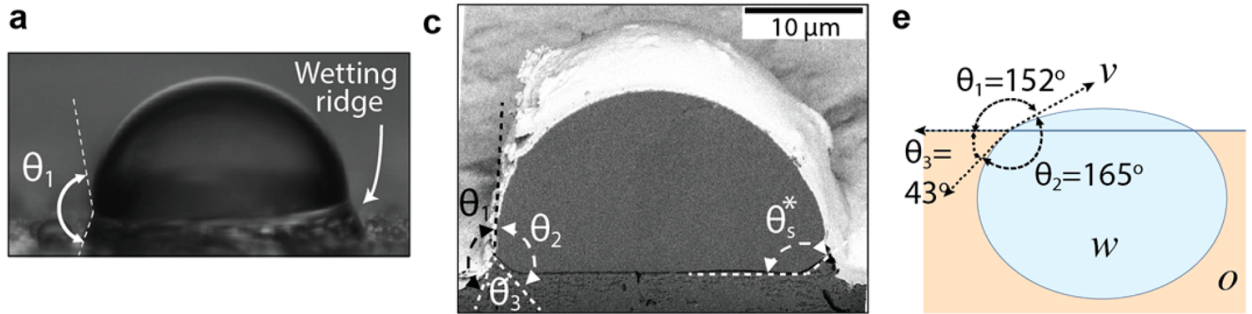

b

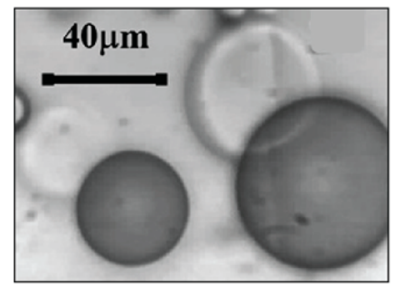

d

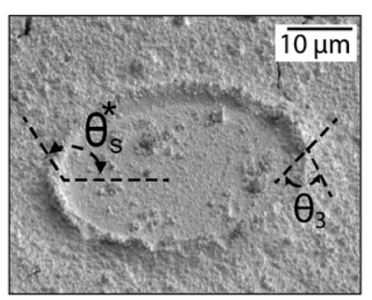

f

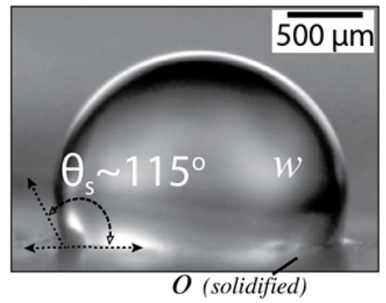

Figure 2. Optical and cryo-FIB-SEM observations of water condensation on cyclohexane and contact angle estimations and measurements. (a) Side view of a $2 \mu \mathrm{L}$ water droplet $\left(T_{\mathrm{a}}=21{ }^{\circ} \mathrm{C}, \mathrm{RH}=27 \%, T_{\mathrm{c}}=1{ }^{\circ} \mathrm{C}\right)$. A distinct wetting ridge of liquid cyclohexane is visible here. This ridge corresponds in (c) and (d) to the cryofreezing of the layer at the drop perimeter. (b) Microscopic image (top view) of a water drop condensed on cyclohexane at $3{ }^{\circ} \mathrm{C}$. The image is taken $20 \mathrm{~ms}$ after the jump of the largest drop. (c) Cryo-FIB-SEM images of a droplet showing the droplet contact line morphology and the estimation of contact angles $\left(\theta_{1,2,3}\right)$ at the triple line of contact (water/vapor/liquid cyclohexane) and the nonequilibrium contact angle $\theta_{\mathrm{s}}^{*}$ (water/solid cyclohexane/liquid cyclohexane). A distinct wetting ridge is always visible. (d) Cryo-FIB image of the cyclohexane surface after droplet detachment during cryofreezing, which corresponds to the nonequilibrium contact angle $\theta_{\mathrm{s}}^{*}$. (e) Schematic of a water droplet immersed in bulk liquid cyclohexane showing the different contact angles at the three-phase contact line. The angles are the same as the contact angles of the water lens on cyclohexane in (a) and (c). (f) Side view of a $10 \mu \mathrm{L}$ water droplet $\left(T_{\mathrm{a}}=21{ }^{\circ} \mathrm{C}, T_{\mathrm{c}}=1{ }^{\circ} \mathrm{C}\right)$ sitting on solid cyclohexane. Note that, unlike the observations in (b) and (c), there is complete absence of a wetting ridge around the droplet, suggesting the absence of cyclohexane melting, and thus, the contact angle displayed by the droplet represents the mean contact angle of water with the solid cyclohexane surface.

droplet on a cyclohexane surface alternating between a complete solid and a partially melted state.

When looking at the condensed water droplets, a wetting ridge is clearly visible at the drop perimeter in the side view (see Figure 2a), even when the drop is immobile. The top view of water droplets shows that a part of this wetting ridge remains adhered to the substrate when the drop moves (Figure $2 b$ ). The presence of the cyclohexane ridge around the water droplets may be a consequence of a balance of interfacial forces were the ridge ${ }^{28,34}$ formed when cyclohexane was in the melted stage. However, such a formation left on the substrate could also be attributed to plastic deformation of solid cyclohexane at the drop perimeter, similar to the case of water drops sitting on deformable soft substrates. $^{35,36}$

The optical images provide useful but limited observations since the topology of the water-cyclohexane interface beneath the droplet is invisible in such images. To observe the hidden topography of the droplet-surface interface, we performed cryo-FIB-SEM measurements of condensed droplets (see section 2.2). The SEM image of the cryo-FIB milled crosssection (Figure 2c) reveals that the water droplet is situated on top of a cyclohexane dish with a flat bottom and raised corners. The profile of the cyclohexane surface further becomes clear in Figure 2d, which shows the imprints left over after a water drop delaminated from the cyclohexane surface. From this image the presence of the $2-3 \mu \mathrm{m}$ tall ridge around the drop perimeter with a flat interface is clearly visible. The absence of any visible distortion beneath the droplet (Figure 2c,d) rules out the possibility of plastic deformation of solid cyclohexane. Note that the drop delamination likely occurs due to thermal expansion mismatch between the frozen drop and the cyclohexane layer during transition from liquid nitrogen slush freezing $\left(-210^{\circ} \mathrm{C}\right)$ to the cryo-FIB-SEM $\left(-160\right.$ to $\left.-192{ }^{\circ} \mathrm{C}\right)$.
Resolving the state (i.e., liquid vs solid) of the cyclohexane within the ridge surrounding the drop before cryopreservation is challenging using SEM as the contrast in backscatter imaging relies mostly on the density difference, but is not sufficiently sensitive to detect the difference between liquid and solid cyclohexane.

From the discussion above, it is clear that the wetting ridge is likely formed as a result of the melting of cyclohexane. The spreading coefficient $\left(S_{\mathrm{ow}(\mathrm{v})}\right)$ of an immiscible oil (o) on a water droplet $(\mathrm{w})$ in the presence of an air environment $(\mathrm{v})$ is given by $S_{\mathrm{ow}(\mathrm{v})}=\sigma_{\mathrm{wv}}-\sigma_{\mathrm{ov}}-\sigma_{\mathrm{wo}}$, where $\sigma_{\mathrm{wv}}$ is the surface tension of water, $\sigma_{\mathrm{ov}}$ is the surface tension of oil, and $\sigma_{\mathrm{wo}}$ is the interfacial tension of oil-water. For the water-liquid cyclohexane system, $\sigma_{\mathrm{wv}}=72.2 \mathrm{mN} / \mathrm{m}, \sigma_{\mathrm{ov}}=25.26 \mathrm{mN} / \mathrm{m}^{21}$ and $\sigma_{\text {ow }}=50.2 \mathrm{mN} / \mathrm{m}^{37}$ at $20^{\circ} \mathrm{C}$, so that the spreading coefficient $\left(S_{\text {ow }(v)}\right)$ of cyclohexane with respect to water is negative, implying that liquid cyclohexane does not cloak the water droplets and forms a distinct three-phase contact line around a droplet that results in formation of the wetting ridge. ${ }^{28}$ Thus, in Figure 2a,b, the material within the ridge likely corresponds to an excess of melted cyclohexane that has not yet frozen between jumps. As the droplet jumps, depending on the closeness of the substrate temperature to $T_{\mathrm{m}}$, the leftover material either evaporates or freezes and sublimates. Far from the $T_{\mathrm{m}}$, the rim left after drop motion rapidly fades away due to evaporation or sublimation. Near the $T_{\mathrm{m}}$, it remains liquid for a long time.

On solid cyclohexane during condensation, since the wetting ridge is due to the melted cyclohexane layer, the shape and morphology of the water droplet near its vicinity are determined by the equilibrium of forces at the contact line of the liquid cyclohexane, water, and air interface. The contact angle of water with the wetting ridge surrounding it can then be 
expected to be similar to the droplet shape profile of water in bulk liquid cyclohexane. On bulk liquid cyclohexane, a water droplet forms a lenticular shape (Figure 2e) since $S_{\mathrm{ow}(\mathrm{v})}<0$. From the balance of interfacial tensions at the three-phase contact line, the different components of the contact angles of this lens are given as $\theta_{1}=151.6^{\circ}, \theta_{2}=165.2^{\circ}$, and $\theta_{3}=43.2^{\circ}$ (see also the Supporting Information). From optical images (Figure 2a), the only visible contact angle is in the vapor phase between liquid cyclohexane and water. It can be estimated to be $\theta_{1}=150^{\circ} \pm 2^{\circ}$ and very closely matches the theoretical estimates. However, the other contact angles $\left(\theta_{2}, \theta_{3}\right)$ cannot be measured from these images. The cryo-FIB-SEM reveals the inner topology of the droplet-cyclohexane interface, and one can thus deduce all the contact angles of liquid water on liquid cyclohexane (Figure 2c). The angles at the top of the rim give the contact angles $\theta_{1,2,3}$ between water, vapor, and liquid cyclohexane as shown in the drop section of Figure 2c. Averaging on several drops gives the following values: $\theta_{1} \approx$ $149^{\circ}, \theta_{2} \approx 148^{\circ}$, and $\theta_{3} \approx 63^{\circ}$. The $\theta_{1}$ value is in excellent agreement with the optical observation, and the theoretical estimates of water with liquid cyclohexane. The contact angles $\theta_{2}$ and $\theta_{3}$ are also within $10 \%$ of the values as obtained from theoretical estimates. This range of error may originate from the uncertainty in measurements due the presence of defects at the triple contact line or the tilt of the cryo-SEM sample during the imaging. In addition to the contact angles between water and liquid cyclohexane at the rim, we also observe that the water droplet forms an apparent contact angle at the base of the rim with the underlying cyclohexane surface, the mean value of which was found to be $\theta_{\mathrm{s}}^{*} \approx 144^{\circ}$ (Figure $2 \mathrm{c}$ ). We refer to this contact angle as the nonequilibrium contact angle $\theta_{s}^{*}$, since the water/liquid/solid cyclohexane contact line corresponds to a nonequilibrium situation where liquid and solid cyclohexane phases are in contact under a temperature gradient. Furthermore, this large value relates to the advancing contact angle as the drop is growing.

As mentioned before, the droplets undergoing motion on cyclohexane are in intermittent contact with the solid and liquid cyclohexane. From the above-mentioned analysis, we can deduce the shape of the droplet in contact with liquid cyclohexane, but the presence of the wetting ridge makes it difficult to ascertain the shape of the droplet in contact with solid cyclohexane. To measure the water droplet contact angle with solid cyclohexane, we deposited an isolated cold water droplet (at $\sim 0-2{ }^{\circ} \mathrm{C}$ ) on solid cyclohexane maintained at a Peltier temperature of $2{ }^{\circ} \mathrm{C}$ (Figure 2f). Condensation on the surface can lead to melting of the top layer, or near the droplet, thereby giving erroneous measurements. To avoid such a problem, the region around the droplet was flushed with dry air to decrease the humidity and thereby prevent condensation. It is clear from Figure $2 \mathrm{f}$ that a wetting ridge is completely absent around the cold water droplet-confirming again the earlier hypothesis that the wetting ridge originates from the melting of cyclohexane. From these experiments we were able to deduce the apparent contact angle $\left(\theta_{s}\right)$ of the water drop with solid cyclohexane (Figure $2 \mathrm{f}$ ) to be nearly $115^{\circ}$.

It is clear from our measurements that the contact angles of water droplets on the liquid cyclohexane $\left(\theta_{2}\right)$ and solid cyclohexane $\left(\theta_{s}\right)$ differ considerably. This large difference in contact angle plays a crucial role along with melting-induced depinning in providing the initial thrust for the droplets to "hop" suddenly. After the droplet becomes depinned, the droplet inertia may sustain the droplet motion until the film underneath the droplet refreezes. However, depending upon the melted region thickness of cyclohexane, the viscous dissipation within this region may also play an important role in governing the dynamics of droplet mobility. The abovementioned aspects related to the droplet propulsion are discussed in the next section.

3.2. Dynamics of Droplet Propulsion. In the classical Leidenfrost problem, the droplet motion is guided by the competition between the evaporation flux from the source (droplet or the surrounding medium) and the Laplace pressure applied on the vapor film that induces Poiseuille flow to the vapor. ${ }^{15,27}$ For a droplet size smaller than the capillary length, a nonwetting droplet in the Leidenfrost state assumes an almost spherical shape because of the absence of any contact line. Compared to the above-mentioned case, the droplet on cyclohexane is held to the surface by the wetting ridge and is intermittently separated from the solid cyclohexane by a melted layer. The droplet on cyclohexane also exerts a Laplace pressure on the melted region; however, the presence of the three-phase contact line provides additional forces that make estimating the melted layer thickness difficult using the classical Leidenfrost effect. The situation is made further complicated because the droplet motion occurs in short bursts (in the usual jumping motion) as the droplet transitions from a freely floating state on a liquid layer to the highly pinned state on the solid cyclohexane. In the discussion below, we provide an estimate of the average melt layer thickness that is achieved before a droplet motion is initiated.

A typical in-plane jumping behavior of a droplet is accompanied by a change in droplet shape as the droplet grows by condensation. To quantitatively show this behavior, we traced the evolution of some randomly chosen droplets on the cyclohexane surface during condensation, and evaluated the evolution of the droplet radius over time (Figure 3). We find

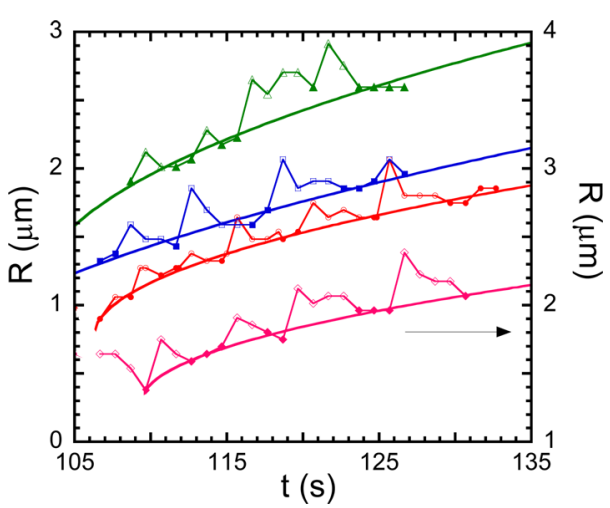

Figure 3. Drop radius evolution (open and full symbols, thin solid lines) between coalescence events under the experimental conditions of ref 19 (four different drops; for clarity, one data set is shifted to the right ordinate). Drop spreading (stage I) and relaxing (stage II) are clearly seen, although the data are taken every $1 \mathrm{~s}$. Full symbols correspond to the drop radius at the end of stage II. The bold solid lines are fits to the growth law $R=R_{0}+k\left(t-t_{0}\right)^{1 / 2}$ (see the text).

that the droplet radius increases overall with time due to condensation; however, the growth behavior is discontinuous, with droplet sizes showing alternately an increase and a decrease in drop size. As the time between each data point is $1 \mathrm{~s}$ and each frame lasts $40 \mathrm{~ms}$, the radius increase is seen with a time resolution of $1 \mathrm{~s}$. The decrease lasts longer, on the order of several seconds. 


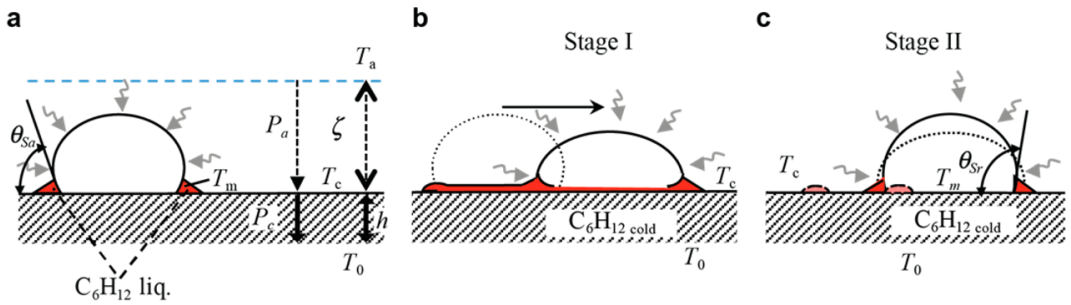

Figure 4. Schematic of the in-plane jumping process. For notations, see the text. (a) Substrate heating process. (b) Stage I, melting, moving, and freezing-induced stopping. Melting of solid cyclohexane occurs at the water-substrate contact area. It produces a liquid film beneath the water drop that completely wets solid cyclohexane. (c) Stage II, relaxation of the contact line.

During the entire droplet growth process, heat is continuously released to the substrate. For a single, isolated drop, the water vapor profile around the drop surface is radially symmetric and the drop radius follows ${ }^{32,38}$

$$
R=k t^{x}
$$

with $x=1 / 2$. Our measurements show that the droplet growth profile on the cyclohexane surface roughly fits this growth curve (Figure 3). The droplet evolution was found to fit $R=R_{0}+k(t$ $\left.-t_{0}\right)^{1 / 2}$ (solid bold lines), where $R_{0}$ and $t_{0}$ correspond to the drop radius value and time after the last coalescence event, and the constant $k$ was estimated as $0.25 \pm 0.1 \mu \mathrm{m} \cdot \mathrm{s}^{-1 / 2}$ from the graph. This exponent $(x=1 / 2)$ contrasts with what is found for usual dropwise condensation (breath figures) where generally droplets are found to grow following the $x=1 / 3$ law. In the usual cases, the drops are close enough such that their water vapor profiles overlap, resulting in a linear concentration gradient perpendicular to the surface and a growth exponent given by $x=1 / 3 .^{38}$ In the present situation, drops are found in general far from each other due to their motion and the vapor regions near the droplets likely do not overlap and hence result in the $x=1 / 2$ law (Figure 3).

Note that, when two drops come into physical contact, either immediate coalescence occurs (within a few milliseconds, which is the time necessary to form and develop a liquid bridge ${ }^{39}$ ) or a composite drop may sometimes form with a separating film of liquid cyclohexane between them. ${ }^{40}$ Although cyclohexane does not cloak the water drops, the wetting ridge around a droplet due to the melted cyclohexane may form a separating film between the droplets, and drainage of this film results in coalescence delay, ${ }^{41-43}$ leading to formation of the composite drop. However, the composite drop behaves as a single drop, and undergoes the same motion (jumping or crawling) as other individual drops (Figure 1e).

As the droplets grow during condensation (Figure 3), the phenomenon of droplet propulsion occurs along the side of the growth. The droplet evolution can be divided to occur in two stages. Stage I comprises melting the substrate due to drop condensation (condensation is described in Figure 4a), resulting in droplet depinning and spreading and movement until freezing of the cyclohexane film (Figure 4b), and stage II comprises relaxation motion of the contact line (i.e., a change in drop contact radius and contact angle) after substrate freezing (Figure 4c).

In the following subsections we aim to evaluate the characteristics of the droplet jumping behavior, i.e., explain the droplet radius evolution shown in Figure 3, the drop jumping length, its velocity, etc. Before discussing the details of the process, we nevertheless need to determine some basic information: substrate heating process, thickness of the melted region below the droplet, etc.
3.2.1. Heating and Melting of the Substrate during Drop Condensation. 3.2.1.1. Bare Substrate and Estimation of the Substrate Temperature near the Solid-Air Interface. The temperature of the substrate at the places where condensation does not take place results from the balance of two opposite heat fluxes, $P_{a}$ corresponding to convective air heating, and $P_{\mathcal{c}}$ the cooling flux from the Peltier thermostat below the cyclohexane layer.

The heat flux $P_{\mathrm{a}}$ occurs within a boundary layer of thickness $\zeta$ and can be written as

$$
\frac{P_{\mathrm{a}}}{S}=\lambda_{\mathrm{a}} \frac{T_{\mathrm{a}}-T_{\mathrm{c}}}{\zeta}
$$

Here $S$ is the surface area of consideration and $\lambda_{\mathrm{a}}$ is the air thermal conductivity. We analyze only the case of natural convection, the situation most often encountered in this study. In the case of natural convection, $\zeta$ can be evaluated by performing a scaling analysis of convection above the horizontal cooled plate. ${ }^{44}$ The hydrodynamic boundary layer is the region where air flows parallel to the plate due to buoyancy effects. Its thickness, $\delta$, is a function ${ }^{44}$ of the Grashof number $G r=$ $g \beta \Delta T L^{3} / \nu^{2}$ through $\delta \approx L\left(G r^{-1 / 5}\right)$. Here $L(\approx 100 \mathrm{~mm})$ is the plate characteristic length, $\Delta T=T_{\mathrm{a}}-T_{\mathrm{c}}(\approx 17 \mathrm{~K}), g=9.81 \mathrm{~m}$. $\mathrm{s}^{-2}$ is the earth's acceleration constant, $\beta$ is the air volumetric thermal expansion coefficient (air is considered an ideal gas, Table 1 ), and $\nu$ is the air kinematic viscosity (Table 1 ). These values give $G r \approx 3 \times 10^{6}$ and $\delta \approx 5 \mathrm{~mm}$. As $\delta \approx \Delta T^{0.2}$, its value is only weakly sensitive to temperature. The maximal velocity in the hydrodynamic layer is given by $U_{\mathrm{m}} \approx(\delta g \beta \Delta T)^{1 / 2} \approx 5 \mathrm{~cm}$. $\mathrm{s}^{-1}$. The thickness $\zeta$ corresponds to the value where heat transport by conduction becomes more efficient than by convection or a Peclet number $P e=U \zeta / D_{\mathrm{T}}<1\left(D_{\mathrm{T}}=2.2 \times\right.$ $10^{-5} \mathrm{~m}^{2} / \mathrm{s}$ is the air thermal diffusivity, ${ }^{45}$ and $U$ is the air velocity). Because the air velocity decreases near the plate following a parabolic flow, $\zeta$ can be deduced from $P e=\left(U_{\mathrm{m}} \zeta\right)$ $\left.D_{\mathrm{T}}\right)(\zeta /(\delta / 2))^{2}=1$. Its value $\zeta=\left[D_{\mathrm{T}} \delta^{3 / 2} /(\beta g \Delta T)^{1 / 2}\right]^{1 / 3} \approx 2.2$ $\mathrm{mm}$. Using this value, we obtain $P_{\mathrm{a}} / S \approx 180 \mathrm{~W} / \mathrm{m}^{2}$.

Heat removal occurs through a solid cyclohexane layer, which has a thickness $h(\sim \mathrm{mm})$. The lower boundary of the cyclohexane layer is maintained at constant temperature $T_{0}(\sim 3$ ${ }^{\circ} \mathrm{C}$ ) by means of a thermostat. The cooling heat flux $P_{\mathrm{c}}$ can thus be written as

$$
\frac{P_{c}}{S}=\lambda_{\mathrm{cs}} \frac{T_{\mathrm{c}}-T_{0}}{h}
$$

Here $\lambda_{\mathrm{cs}}$ is the solid cyclohexane thermal conductivity (Table $1)$. No temperature changes are observed during the experiment time scale. One thus assumes a quasi steady state and equates the heating and cooling loads $P_{\mathrm{a}}=P_{\mathrm{c}}$ (only the case 
without condensation is considered here). With $h \approx 1.5 \mathrm{~mm}$, one obtains the realistic value $T_{\mathrm{c}}-T_{0} \approx 1.3 \mathrm{~K}$.

3.2.1.2. Substrate with a Residing Drop. Let us now consider a drop that has just moved to its new position. Its temperature at contact with the substrate, initially at temperature $T_{\mathcal{c}}$ results from the balance between $P_{a}, P_{\mathcal{c}}$ the water condensation process corresponding to the latent heat production $P_{L_{\mathrm{w}}}$, and, when cyclohexane melting occurs, the cyclohexane melting latent heat $P_{L_{c}}$. Latent heat flux can be written as

$$
P_{L_{i}}=\rho_{i} L_{i} \frac{\mathrm{d} V_{i}}{\mathrm{~d} t}
$$

with $i$ standing for water $(\mathrm{w})$ or cyclohexane (c). The other parameters are (Table 1$)$ the density of liquid water $\left(\rho_{\mathrm{w}}\right)$, the density of liquid cyclohexane $\left(\rho_{\text {lc }}\right)$, the latent heat of water condensation $\left(L_{\mathrm{w}}\right)$, and the latent heat of cyclohexane melting $\left(L_{\mathrm{c}}\right)$.

The volume evolution $V_{\mathrm{w}}=\pi F(\theta) R^{3}$ of a water drop where condensation proceeds on its surface with constant contact angle $\theta$ is the derivative

$$
\frac{\mathrm{d} V_{\mathrm{w}}}{\mathrm{d} t}=3 \pi \mathrm{F}(\theta) R^{2} \frac{\mathrm{d} R}{\mathrm{~d} t}
$$

Here $F(\theta)=\left(2-3 \cos \theta+\cos \theta^{3}\right) /\left(3 \sin \theta^{3}\right)$ is the volume geometrical factor. As the contact line of the droplet is pinned on the substrate, the contact angle varies during growth, from the receding to the advancing value, causing the water vapor profile to vary. However, this variation is weak as the contact angle is near $90^{\circ}$. We will thus neglect this variation in the following for the sake of simplification.

From eq 4 , since, $R=k t^{1 / 2}$, the volume evolution can be written as

$$
\frac{\mathrm{d} V_{\mathrm{w}}}{\mathrm{d} t}=\frac{3}{2} \pi F(\theta) k^{2} R
$$

At the same time, the melting of a cyclohexane layer of thickness $e$ under the drop corresponds to the volume $V_{\mathrm{c}}=$ $\pi e R^{2}$.

3.2.1.3. Melting Time. In the classical Leidenfrost problem, the vapor layer prevents the droplet from boiling, and the droplet is maintained at nearly the boiling temperature. Similarly, in the case of a condensed droplet on the cyclohexane surface, if the process of stick/slip is in a steady state, the water drop temperature remains at the melting temperature of cyclohexane $T_{\mathrm{m}}$. In the time $t_{\mathrm{m}}$, condensation only heats the substrate beneath the drop from $T_{\mathrm{c}}$ to $T_{\mathrm{m}}$, corresponding to the energy $Q_{0}$ and then melts it, corresponding to the energy $P_{L_{c}} t_{\mathrm{m}}$. As a result, the time $t_{\mathrm{m}}$ required to heat and melt a solid cyclohexane layer of thickness $e$ corresponds to an energy balance where heating loads (air, $P_{\mathrm{a}} t_{\mathrm{m}}$; water condensation, $P_{L_{w}} t_{\mathrm{m}}$ ) and cooling loads (Peltier, $P_{c} t_{\mathrm{m}} ;$ cyclohexane solidification, $P_{L_{\mathrm{c}}} t_{\mathrm{m}}$; sensitive heat of solid cyclohexane from $T_{\mathrm{c}}$ to $T_{\mathrm{m}}, Q_{\mathrm{c}}$ ) enter. One thus obtains the following equation, where heating and cooling energies are equalized:

$$
\left(P_{\mathrm{a}}+P_{L_{\mathrm{w}}}\right) t_{\mathrm{m}}=Q_{\mathrm{c}}+\left(P_{\mathrm{c}}+P_{L_{\mathrm{c}}}\right) t_{\mathrm{m}}
$$

In this equation we have split the contributions just without condensation and phase changes, corresponding to $P_{\mathrm{a}}=P_{\mathcal{c}}$ and the other condensation-induced terms. $Q_{c}$ is the sensitive heat of the cyclohexane layer: 

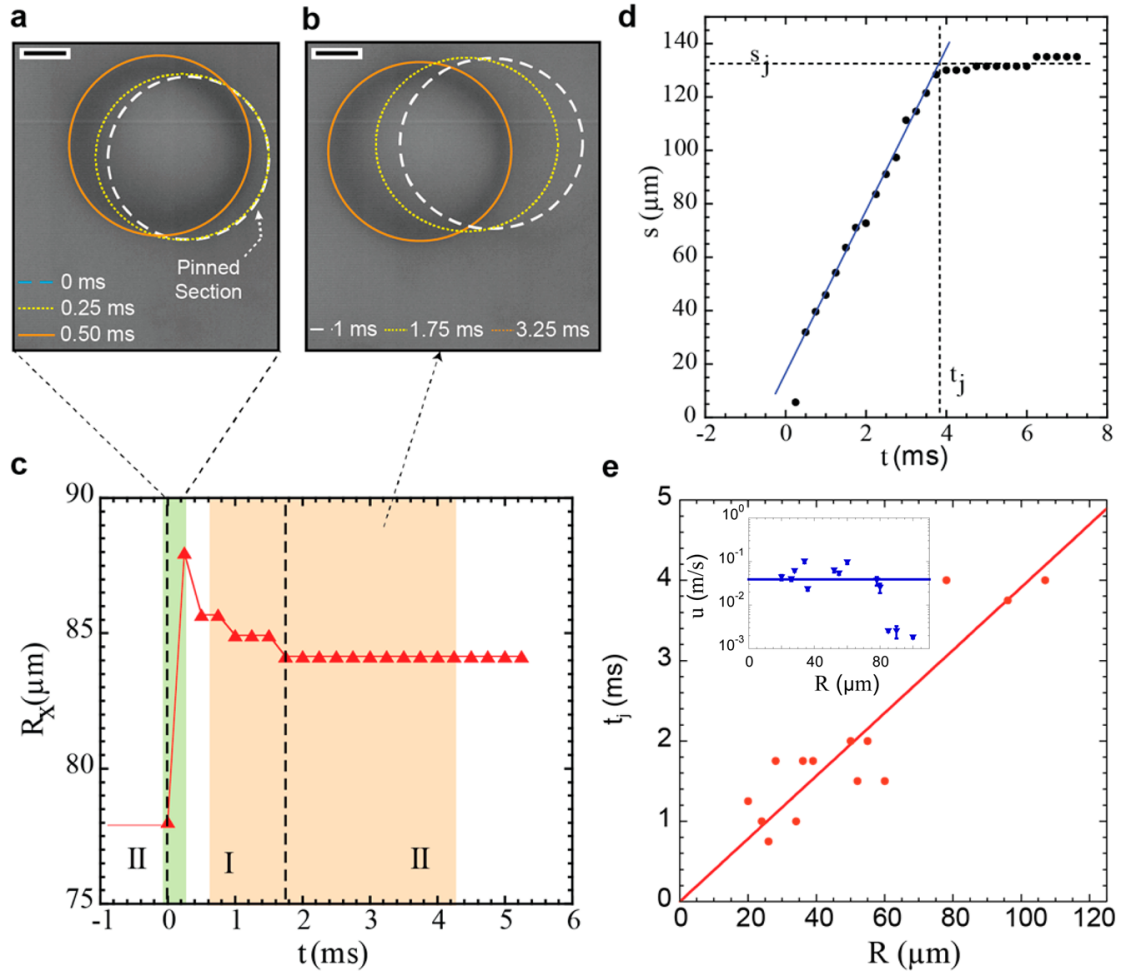

Figure 5. Examples of (a) asymmetric drop depinning and spreading on cyclohexane $\left(T_{\mathrm{a}}=23{ }^{\circ} \mathrm{C}, \mathrm{RH}=100 \%, T_{\mathrm{c}}=3{ }^{\circ} \mathrm{C}\right.$, flux $\left.0.2 \mathrm{~L} / \mathrm{min}\right)$ and $(\mathrm{b})$ jumping motion displayed after the asymmetric drop depinning behavior ends. The scale bar in (a) and (b) corresponds to $20 \mu \mathrm{m}$. (c) Evolution at short time of the drop-projected radius. (d) Evolution of the distance $s$ traveled by the drop in (b) showing a constant velocity before brusquely stopping at $s=s_{\mathrm{j}}$ and $t=t_{\mathrm{j}}$. (e) Variation of $t_{\mathrm{j}}$ with respect to the drop radius $R$. The line is a fit with slope $\gamma=39 \pm 2 \mathrm{~s} \cdot \mathrm{m}^{-1}$. Inset: velocity $u$ with respect to the drop radius. Error bars are measurement uncertainties.

$$
Q_{\mathrm{c}}=\pi e R^{2} \rho_{\mathrm{cs}} C_{p, \mathrm{cs}}\left(T_{\mathrm{m}}-T_{\mathrm{c}}\right)
$$

where $C_{p, c s}$ is the specific heat of solid cyclohexane (Table 1 ). As $P_{\mathrm{a}}=P_{\mathrm{c}}, P_{L_{\mathrm{c}}} t_{\mathrm{m}}=\rho_{\mathrm{cs}} \pi R^{2} e L_{\mathrm{c}}$, and $P_{L_{\mathrm{w}}}=\rho_{\mathrm{w}} L_{\mathrm{w}}\left(\mathrm{d} V_{\mathrm{w}} / \mathrm{d} t\right)$, eq 1 is recovered. The proportionality constant is equal to

$$
\alpha=\frac{2}{3} \frac{\rho_{\mathrm{cs}}}{\rho_{\mathrm{w}}} \frac{L_{\mathrm{c}}+C_{p, \mathrm{cs}}\left(T_{\mathrm{m}}-T_{\mathrm{c}}\right)}{L_{\mathrm{w}}} \frac{e}{k^{2} F(\theta)}
$$

$k^{2}$ and $\alpha$ can be measured experimentally, enabling calculation of a typical melted layer thickness. Under the conditions of ref $19, \alpha=6 \times 10^{4} \mathrm{~s} \cdot \mathrm{m}^{-1}, k^{2} \approx 6.25 \times 10^{-14} \mathrm{~m}^{2} \cdot \mathrm{s}^{-1}$ (Figure 3), and $T_{\mathrm{m}}-T_{\mathrm{c}} \approx 3 \mathrm{~K}$. The volume of the drop can be estimated as that of a half-sphere (see Figure 2), with $F\left(90^{\circ}\right)=2 / 3$. Using these values, we finally obtain the thickness of the melt region, $e$ $\approx 300 \mathrm{~nm}$. Note that this is the mean thickness of the melted layer. The constant droplet velocity (Figure $5 \mathrm{~d}$, section 3.2.2) prior to the pinning of the droplet implies that the thickness of the melted layer remains nearly constant. The depinning to pinning transition exhibited during the jumping motion of the droplet occurs because the temperature of the melted layer progressively decreases until the layer is frozen within a very short time interval lasting a frame $(t<0.25 \mathrm{~ms})$.

3.2.2. Stage I: Droplet Movement until Freezing of the Cyclohexane Film. Figure 5a shows typical drop motion just as droplet depinning is initiated. The initial time $(t=0)$ is determined within $250 \mu \mathrm{s}$. The change from stage II, where the drop is pinned to solid cyclohexane, to stage I, which corresponds to the drop lying on liquid cyclohexane, occurs in less than one frame $(250 \mu \mathrm{s})$. The drop spreads (i.e., its radius increases) and starts moving in the same direction as it is spreading. This observation corresponds to the process where the water drop, pinned to solid cyclohexane, grows. Due to the pinning, the contact angle of water/solid cyclohexane increases toward the advancing contact angle, $\theta_{\mathrm{s}, \mathrm{adv}}$. When melting occurs, the drop shape changes to have only one triple line with liquid cyclohexane. Because depinning due to random thermal and substrate inhomogeneities does not occur at the same time on the perimeter, spreading is not symmetric as can be seen in Figure 5a.

In stage I the drop moves rapidly on a layer of molten cyclohexane. This period lasts typically a few milliseconds. When the drop resides on the liquid layer, its perimeter is not pinned anymore and it moves freely at constant velocity until the layer begins to refreeze. At that point, viscous dissipation increases dramatically, leading to termination of drop motion. The motion starts when the drop shape changes (the drop spreads) as cyclohexane melts. Melting cannot be exactly symmetric, due to small random inhomogeneities in temperature and substrate properties. The asymmetry results in a capillary force $f \approx 2 \pi \sigma_{\mathrm{wv}} R$ that deforms the drop in a preferred direction (Figure 5a). Here $\sigma_{\mathrm{wv}}$ refers to the surface tension of water in air. This force gives an initial velocity $u_{0}$ to the drop, which decreases with time because of the friction in the film. Motion will stop after a length $L_{\mathcal{c}}$ which can be estimated by equating the drop kinetic energy, ${ }^{1}{ }_{2} \rho_{\mathrm{w}} V_{\mathrm{w}} u_{0}{ }^{2}$, and film viscous dissipation, $\left(\rho_{\mathrm{w}} \nu u_{0} R^{2} / e\right) L_{\mathcal{c}}$ with $\nu$ the kinematic viscosity of water $\left(=1.15 \times 10^{-6} \mathrm{~m}^{2} \cdot \mathrm{s}^{-1}\right.$; see Table 1$)$. One obtains $L_{\mathrm{c}} \approx$ $e R u_{0} / \nu \approx 1 \mu \mathrm{m}$ for a drop radius of $\sim 80 \mu \mathrm{m}$ with typical velocity $u_{0}=u=0.04 \mathrm{~m} \cdot \mathrm{s}^{-1}$ (Figure $5 \mathrm{~d}$ ).

The capillary force can thus contribute only little to the motion, which must be ensured by another, steady force. As 
temperature gradients are present at the surface of the drop, at the liquid cyclohexane ridge around the drop, and at the interface drop-liquid cyclohexane beneath, thermocapillary effects do exist. Liquid motion in the drop and in the ridge can indeed be observed during condensation (see Supplementary Video S4, Supporting Information). Due to the change of position of the drop after cyclohexane melting, nonsymmetric temperature gradients appear that move the drop until the film freezes toward the cold end, that is, outside the drop. It is, however, very difficult to model these temperature gradients and their interactions with the liquid cyclohexane, water, and air interface. In the simplest case where thermocapillary motion is assumed to be dominated by the interfaces air-water and airliquid cyclohexane, the typical fluid velocity at the interface of the liquid layer (thickness $h$, shear viscosity $\eta$, surface tension derivative $\mathrm{d} \sigma / d T)$ that is submitted to a transverse thermal gradient $(\mathrm{d} T / \mathrm{d} x)$ can be expressed as ${ }^{46}$

$$
u=\frac{h}{4 \eta} \frac{\mathrm{d} \sigma}{\mathrm{d} T} \frac{\mathrm{d} T}{\mathrm{~d} x} \approx \frac{1}{4 \eta} \frac{\mathrm{d} \sigma}{\mathrm{d} T}\left(T_{\mathrm{a}}-T_{\mathrm{m}}\right)
$$

The approximate formulation stems from the scaling assumption that the characteristic lengths for the film and ridge are the same as the typical length scale in the temperature gradient. Typical temperatures for water and liquid cyclohexane are taken as $T_{\mathrm{a}}$ and $T_{\mathrm{m}}$. With $\mathrm{d} \sigma_{\mathrm{ow}} / \mathrm{d} T \approx-1.4 \times 10^{-4} \mathrm{~N} \cdot \mathrm{m}^{-1}$. $\mathrm{K}^{-121}$ and $\mathrm{d} \sigma_{\text {ov }} / \mathrm{d} T \approx-0.53 \times 10^{-3} \mathrm{~N} \cdot \mathrm{m}^{-1} \cdot \mathrm{K}^{-1}, 47$ typical velocity values are $\sim-1 \mathrm{~ms}^{-1}$, corresponding to a motion toward the coldest areas, outside the drop. Note that eq 13 is a dimensional equation. A precise value would need a much more detailed analysis that is beyond the scope of the present study.

When freezing of the liquid cyclohexane does not occur, a steady motion triggered by these thermocapillary forces occurs: it is the crawling motion. Freezing is hampered by a too large film thickness; this is, e.g., the case when two drops collide as in Figure $1 b$, where drops merge their lubrication layers.

We note that the measured droplet velocity here is at least an order of magnitude smaller as compared to droplet velocities ${ }^{27}$ in the classical Leidenfrost problem. This is not surprising because the viscous dissipation in the underlying liquid is significantly larger than dissipation in the vapor.

Figure $5 \mathrm{c}$ represents the evolution of the distance $s$ traveled by one drop. The constant slope of the curve in Figure $5 \mathrm{~d}$ confirms a constant drop velocity until its sudden stop at time $t$ $=t_{\mathrm{j}}$. The velocity can be directly calculated as $u=s_{\mathrm{j}} / t_{\mathrm{j}}$, with $s_{\mathrm{j}}$ being the distance traveled by the drop. The distance $s_{\mathrm{j}}$ is proportional to $R$ following eq 2 . In the present experiments, a proportionality constant of $\beta=1.6 \pm 0.1$ was measured. As $t_{\mathrm{j}}$ is also proportional to $R$ (Figure $5 \mathrm{e}$ )

$$
t_{\mathrm{j}}=\gamma R
$$

one deduces that the velocity is constant with respect to $R$, in agreement with eq 13. A plot of $u=s_{\mathrm{j}} / t_{\mathrm{j}}$ is reported in the inset of Figure 5e. Large drops show a lower velocity that can be attributed to local inhomogeneities; however, no clear variation of $u$ with $R$ is visible. The mean value $u=(3.8 \pm 0.9) \times 10^{-2} \mathrm{~m}$. $\mathrm{s}^{-1}$ is in accord with the ratio $u=\beta / \gamma=(3.8 \pm 0.7) \times 10^{-2} \mathrm{~m}$. $\mathrm{s}^{-1}$. This value is, however, much smaller than the value from eq 13 (a few meters per second). This is not surprising since, as already noted, eq 13 is only dimensional.

The fact that the drop stops after having traveled on $s_{\mathrm{j}} \approx 1.5 R$ can be explained as follows: The distance $1.5 R$ corresponds to having the drop surface nearly entirely on the bare, nonheated surface beside the initial position of the drop. It is in agreement with a near instantaneous freezing process when the drop departs significantly from its initial position.

The time $t_{\mathrm{j}}$ required to freeze this layer can be estimated as follows: While the drop is moving on the substrate, cooling of the drop and layer beneath is performed by the substrate, whose surface is at temperature $T_{c}$. The lower part of the layer is at $T_{\mathrm{c}}$ and its upper part at $T_{\mathrm{m}}$. Both the layer and drop are close to the same temperature $T_{\mathrm{m}}$. This means that only the latent heat of cyclohexane is removed, the freezing process being at constant temperature. It also means that the surface temperature of solid cyclohexane has to adapt to $T_{\mathrm{m}}$ by a thermal diffuse boundary layer of extent $\xi \approx\left(D_{\mathrm{T}, \mathrm{cs}} t\right)^{1 / 2}\left(D_{\mathrm{T}, \mathrm{cs}}=\right.$ $\lambda_{\mathrm{cs}} / \rho_{\mathrm{cs}} C_{p, \mathrm{cs}}$ is the solid cyclohexane thermal diffusivity). Freezing of the liquid layer will terminate at time $t_{j}$ (corresponding to the thermal boundary layer $\xi_{j}$ ) when the sensitive heat gained by the diffuse boundary layer is equal to the latent heat needed for freezing. With $\rho_{\mathrm{cl}}\left(\rho_{\mathrm{cs}}\right)$ the density of liquid (solid) cyclohexane, $C_{p, c s}$ the specific heat of solid cyclohexane, and $S_{c}$ the surface of contact of the liquid layer, the energy $Q$ needed to freeze a liquid layer of thickness $e$ is

$$
\frac{Q}{S_{\mathrm{c}}}=\rho_{\mathrm{cs}} C_{p, \mathrm{cs}}\left[\left(T_{\mathrm{m}}-T_{\mathrm{c}}\right)\right] \xi_{\mathrm{j}}=\rho_{\mathrm{cl}} L_{\mathrm{c}} e
$$

or

$$
t_{\mathrm{j}}=\left[\frac{\rho_{\mathrm{cl}}}{\rho_{\mathrm{cs}}} \frac{L_{\mathrm{c}}}{C_{p, \mathrm{cs}}\left(T_{\mathrm{m}}-T_{\mathrm{c}}\right)}\right]^{2} \frac{e^{2}}{D_{\mathrm{T}, \mathrm{cs}}}
$$

With the values of Table 1 and $T_{\mathrm{m}}-T_{\mathrm{c}} \approx 3 \mathrm{~K}$ (see section 3.2.1.3), a time $t_{\mathrm{j}} \approx 30 \mu \mathrm{s}$ for $e \approx 300 \mathrm{~nm}$ is obtained. The short time scale guarantees near instantaneous freezing of the melted cyclohexane after the drop's departure, and thus explains the presence of ridgelike features (Figure $2 \mathrm{~b}, \mathrm{~d}$ ) that are left behind as the droplet moves to a new location.

3.2.3. Stage II: Relaxation of the Contact Line. After the drop has moved, the liquid imprint of its previous position fades gradually. The imprint corresponds to the residues of the wetting ridge around the drop. This ridge is initially liquid and freezes after some time. It disappears by liquid evaporation (when liquid) and sublimation (when solid). The drop radius slowly diminishes to ideally recover its initial value on solid cyclohexane, with a small increase due to condensation. This process corresponds to the general situation of the motion of the contact line of a drop on a solid. It has been studied for the relaxation of the shape of the composite drop formed after the coalescence of two drops. ${ }^{48}$ Coalescence indeed leads to the formation of a drop whose contact angle is smaller than at equilibrium, as is the case here for the drop when the film beneath freezes. The relaxation process of this nonequilibrium shape is extremely slow due to the enhanced dissipation at the contact line location. It follows an exponential decay with typical time

$$
t_{\mathrm{c}}=K\left(\frac{\eta}{\sigma}\right) R
$$

The parameter $K$ is in the range of $10^{6}-10^{7} .^{48}$ The surface tension term $\sigma$ in eq 17 corresponds to the water surface tension $\left(\sigma_{\mathrm{wv}}\right)$. In the present experiments where the situation is similar, the evolution of the drop radius follows an exponential decay (eq 3 and Figure 6a) corresponding to the relaxation of the contact angle of water/solid cyclohexane, $\theta_{\text {s, }}$ toward the 

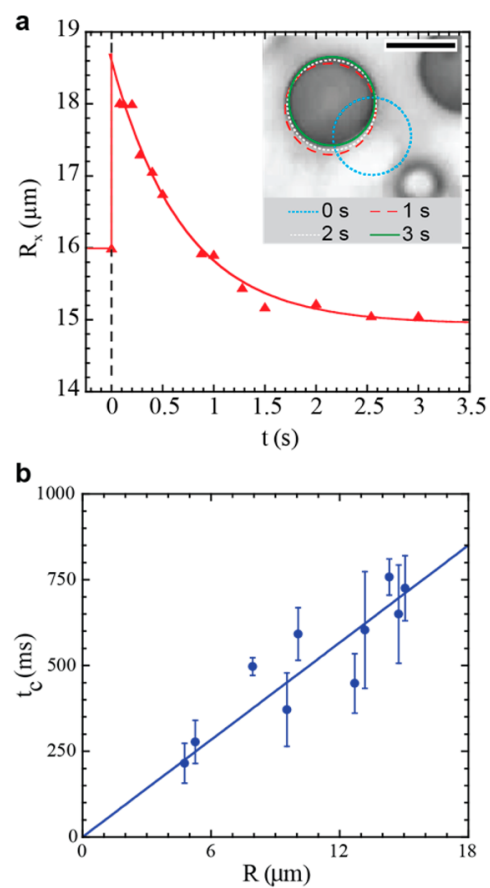

Figure 6. (a) Long times, stage II during freezing $(R=16 \mu \mathrm{m})$. The curve is a fit to eq 3 with $t_{\mathrm{c}}=730 \pm 100 \mathrm{~ms}$. The data at $t=0 \mathrm{~s}$ correspond to the drop radius just before stick/slip. The differences between radius initial values and relaxed values are due to more or less completion of relaxation between jumps. The inset image shows the droplet motion and relaxation as represented in the graph. The scale bar in the inset image corresponds to $30 \mu \mathrm{m}$. (b) Variation of the drop size relaxation time $t_{\mathrm{c}}$ with respect to the drop radius. The line is a fit to eq 17 , which shows proportionality between $t_{\mathrm{c}}$ and $R$.

receding value, $\theta_{\text {s,rec }}$ Note that a difference exists between the initial radius (before jump) and final radius (relaxed value). This is because the drop is always in a jumping motion. Depending on the time between two successive jumps (which varies for every jump), the relaxation can be more or less completed. As expected from eq $17, t_{\mathrm{c}}$ is proportional to $R$ (Figure 6b). The data fit well to eq 17 with $K=3.2 \times 10^{6}$, a value in accord with reference 48 data.

\section{CONCLUDING REMARKS}

The Leidenfrost effect of a liquid drop on a hot plate corresponds to the vapor production below the drop. A film forms, allowing the drops to move erratically. In the present situation, the process is similar but inverted, heat coming from condensation melting the substrate and creating a liquid lubrication film. Drop pinning is thus suppressed, and the drop can freely move under the action of weak forces such as the change of drop form when the drop is depinned and thermocapillary forces. The film below the drop is thin enough such that when the drop moves to the nearest cold area the melted layer freezes nearly instantaneously. This is thus a recurring process characterized by successive time periods of substrate melting, drop motion, substrate freezing, and so on. This process therefore corresponds to a discontinuous, recurring inverted Leidenfrost effect. In some cases, due to drop collisions or geometric defects in the substrate, the layer becomes larger and cannot freeze rapidly. It results in so-called "crawling" motion, characterized by a continuous motion occurring over larger time scales compared to the in-plane "jumping" motion. The Leidenfrost phenomenon stops when the vapor production has ended. Similar to this phenomenon, droplet motion on a PCM such as cyclohexane is not steady and stops when the melted layer freezes. Such "inverted Leidenfrost" types of motion have been observed in other liquids such as benzene ${ }^{20}$ and may be quite general. Furthermore, phase change materials that may have a low vapor pressure could be incorporated within solid textures to form lubricant-impregnated surfaces (LISs). ${ }^{34,49}$ Use of such materials (either in bulk form or in LISs) could be useful for condensation applications, because self-propelled droplets rapidly sweep the surface, thereby resulting in enhanced coalescence and enhanced heat transfer.

\section{ASSOCIATED CONTENT}

\section{Supporting Information}

Description of the contact angles and spreading coefficients of a water droplet in cyclohexane and videos showing the side view (Supplementary Video S2, $0.77 \mathrm{fps)}$ ) and top view (Supplementary Videos S3, 1 fps and S5, $40 \mathrm{fps}$ ) of water droplets condensing on cyclohexane and the top view of the internal flow within a condensing droplet due to thermocapillary forces (Supplementary Video S4, $1 \mathrm{fps}$ ). This material is available free of charge via the Internet at http://pubs.acs.org.

\section{AUTHOR INFORMATION}

\section{Corresponding Author}

*E-mail: daniel.beysens@espci.fr. Phone: +33140795806. Fax: +33140794522 .

\section{Notes}

The authors declare no competing financial interest.

\section{ACKNOWLEDGMENTS}

We acknowledge support from the MIT International Science and Technology Initiative and the MIT-France Program. This work was also partly supported by the Spanish MEC (Grant FIS2011-24642). S.A. thanks the Society in Science-Branco Weiss Fellowship for support. R.N. acknowledges the support of a Marie Curie International Incoming Fellowship (MCIIF) within the seventh European Community Framework Programme.

\section{REFERENCES}

(1) Boreyko, J. B.; Chen, C. H. Self-propelled dropwise condensate on superhydrophobic surfaces. Phys. Rev. Lett. 2009, 103 (18), 184501.

(2) Malvadkar, N. A.; Hancock, M. J.; Sekeroglu, K.; Dressick, W. J.; Demirel, M. C. An engineered anisotropic nanofilm with unidirectional wetting properties. Nat. Mater. 2010, 9 (12), 1023-1028.

(3) Sekeroglu, K.; Gurkan, U. A.; Demirci, U.; Demirel, M. C. Transport of a soft cargo on a nanoscale ratchet. Appl. Phys. Lett. 2011, 99 (6), 063703.

(4) Ju, J.; Xiao, K.; Yao, X.; Bai, H.; Jiang, L. Bioinspired conical copper wire with gradient wettability for continuous and efficient fog collection. Adv. Mater. 2013, 25 (41), 5937-5942.

(5) Daniel, S.; Chaudhury, M. K.; De Gennes, P. G. Vibrationactuated drop motion on surfaces for batch microfluidic processes. Langmuir 2005, 21 (9), 4240-4248.

(6) Boreyko, J. B.; Chen, C. H. Restoring superhydrophobicity of lotus leaves with vibration-induced dewetting. Phys. Rev. Lett. 2009, 103 (17), 174502.

(7) Noblin, X.; Kofman, R.; Celestini, F. Ratchetlike motion of a shaken drop. Phys. Rev. Lett. 2009, 102 (19), 194504.

(8) Ichimura, K.; Oh, S. K.; Nakagawa, M. Light-driven motion of liquids on a photoresponsive surface. Science 2000, 288 (5471), 16241626. 
(9) Florea, L.; Wagner, K.; Wagner, P.; Wallace, G. G.; Benito-Lopez, F.; Officer, D. L.; Diamond, D. Photo-chemopropulsion-Lightstimulated movement of microdroplets. Adv. Mater. 2014, 26 (43), 7339-7345.

(10) Mirsaidov, U. M.; Zheng, H.; Bhattacharya, D.; Casana, Y.; Matsudaira, P. Direct observation of stick-slip movements of water nanodroplets induced by an electron beam. Proc. Natl. Acad. Sci. U.S.A. 2012, 109 (19), 7187-7190.

(11) Foresti, D.; Nabavi, M.; Klingauf, M.; Ferrari, A.; Poulikakos, D. Acoustophoretic contactless transport and handling of matter in air. Proc. Natl. Acad. Sci. U.S.A. 2013, 110 (31), 12549-12554.

(12) Khalil, K. S.; Mahmoudi, S. R.; Abu-Dheir, N.; Varanasi, K. K. Active surfaces: Ferrofluid-impregnated surfaces for active manipulation of droplets. Appl. Phys. Lett. 2014, 105 (4), 041604.

(13) Zhu, Y.; Antao, D. S.; Xiao, R.; Wang, E. N. Real-time manipulation with magnetically tunable structures. Adv. Mater. 2014, 26 (37), 6442-6446.

(14) Longley, J. E.; Dooley, E.; Givler, D. M.; Napier Iii, W. J.; Chaudhury, M. K.; Daniel, S. Drop motion induced by repeated stretching and relaxation on a gradient surface with hysteresis. Langmuir 2012, 28 (39), 13912-13918.

(15) Biance, A. L.; Clanet, C.; Quéré, D. Leidenfrost drops. Phys. Fluids 2003, 15 (6), 1632-1637.

(16) Linke, H.; Alemán, B. J.; Melling, L. D.; Taormina, M. J.; Francis, M. J.; Dow-Hygelund, C. C.; Narayanan, V.; Taylor, R. P.; Stout, A. Self-propelled Leidenfrost droplets. Phys. Rev. Lett. 2006, 96 (15), 154502.

(17) Tersoff, J.; Jesson, D. E.; Tang, W. X. Running droplets of gallium from evaporation of gallium arsenide. Science 2009, 324 (5924), 236-238.

(18) Hilner, E.; Zakharov, A. A.; Schulte, K.; Kratzer, P.; Andersen, J. N.; Lundgren, E.; Mikkelsen, A. Ordering of the nanoscale step morphology as a mechanism for droplet self-propulsion. Nano Lett. 2009, 9 (7), 2710-2714.

(19) Steyer, A.; Guenoun, P.; Beysens, D. Spontaneous jumps of a droplet. Phys. Rev. Lett. 1992, 68 (1), 64-66.

(20) Narhe, R. D.; Khandkar, M. D.; Shelke, P. B.; Limaye, A. V.; Beysens, D. A. Condensation-induced jumping water drops. Phys. Rev. E 2009, 80 (3), 031604.

(21) Yaws, C. L. Yaws' Handbook of Thermodynamic and Physical Properties of Chemical Compounds; Knovel: Norwich, NY, 2003.

(22) Hall, R. S.; Board, S. J.; Clare, A. J.; Duffey, R. B.; Playle, T. S.; Poole, D. H. Inverse Leidenfrost phenomenon. Nature 1969, 224 (5216), 266-267.

(23) Song, Y. S.; Adler, D.; Xu, F.; Kayaalp, E.; Nureddin, A.; Anchan, R. M.; Maas, R. L.; Demirci, U. Vitrification and levitation of a liquid droplet on liquid nitrogen. Proc. Natl. Acad. Sci. U.S.A. 2010, 107 (10), $4596-4600$.

(24) Vakarelski, I. U.; Patankar, N. A.; Marston, J. O.; Chan, D. Y. C.; Thoroddsen, S. T. Stabilization of Leidenfrost vapour layer by textured superhydrophobic surfaces. Nature 2012, 489 (7415), 274-277.

(25) Anand, S.; Varanasi, K. K. Articles and methods for levitating liquids on surfaces, and devices incorporating the same. WO 2013188702 A1. 2013.

(26) Antonini, C.; Bernagozzi, I.; Jung, S.; Poulikakos, D.; Marengo, M. Water drops dancing on ice: How sublimation leads to drop rebound. Phys. Rev. Lett. 2013, 111 (1), 014501.

(27) Quéré, D. Leidenfrost dynamics. Annu. Rev. Fluid Mech. 2013, $45,197-215$.

(28) Smith, J. D.; Dhiman, R; Anand, S.; Reza-Garduno, E.; Cohen, R. E.; McKinley, G. H.; Varanasi, K. K. Droplet mobility on lubricantimpregnated surfaces. Soft Matter 2013, 9 (6), 1772-1780.

(29) Katz, J. I. Jumping droplets. Phys. Rev. E 1994, 49 (1), 914-915.

(30) Abràmoff, M. D.; Magalhães, P. J.; Ram, S. J. Image processing with ImageJ. Biophotonics Int. 2004, 11 (7), 36-41.

(31) Rykaczewski, K.; Landin, T.; Walker, M. L.; Scott, J. H. J.; Varanasi, K. K. Direct imaging of complex nano- to microscale interfaces involving solid, liquid, and gas phases. ACS Nano 2012, 6 (10), 9326-9334.
(32) Anand, S.; Rykaczewski, K.; Subramanyam, S. B.; Beysens, D.; Varanasi, K. K. How droplets nucleate and grow on liquids and liquid impregnated surfaces. Soft Matter 2015, 11 (1), 69-80.

(33) Rykaczewski, K.; Anand, S.; Subramanyam, S. B.; Varanasi, K. K. Mechanism of frost formation on lubricant-impregnated surfaces. Langmuir 2013, 29 (17), 5230-5238.

(34) Anand, S.; Paxson, A. T.; Dhiman, R.; Smith, J. D.; Varanasi, K. $\mathrm{K}$. Enhanced condensation on lubricant-impregnated nanotextured surfaces. ACS Nano 2012, 6 (11), 10122-10129.

(35) Style, R. W.; Dufresne, E. R. Static wetting on deformable substrates, from liquids to soft solids. Soft Matter 2012, 8 (27), 71777184.

(36) Style, R. W.; Boltyanskiy, R.; Che, Y.; Wettlaufer, J. S.; Wilen, L. A.; Dufresne, E. R. Universal deformation of soft substrates near a contact line and the direct measurement of solid surface stresses. Phys. Rev. Lett. 2013, 110 (6), 066103.

(37) Demond, A. H.; Lindner, A. S. Estimation of interfacial tension between organic liquids and water. Environ. Sci. Technol. 1993, 27 (12), 2318-2331.

(38) Sokuler, M.; Auernhammer, G. K.; Liu, C. J.; Bonaccurso, E.; Butt, H. J. Dynamics of condensation and evaporation: Effect of interdrop spacing. Europhys. Lett. 2010, 89 (3), 36004.

(39) Narhe, R. D.; Beysens, D. A.; Pomeau, Y. Dynamic drying in the early-stage coalescence of droplets sitting on a plate. Europhys. Lett. 2008, 81 (4), 46002.

(40) Guadarrama-Cetina, J.; González-Viñas, W. Breath figures of two immiscible substances on a repellent surface. Phys. Rev. E 2013, 87 (5), 054401.

(41) Eggers, J.; Lister, J. R.; Stone, H. A. Coalescence of liquid drops. J. Fluid Mech. 1999, 401, 293-310.

(42) Chan, D. Y. C.; Klaseboer, E.; Manica, R. Film drainage and coalescence between deformable drops and bubbles. Soft Matter 2011, 7 (6), 2235-2264.

(43) Boreyko, J. B.; Polizos, G.; Datskos, P. G.; Sarles, S. A.; Collier, C. P. Air-stable droplet interface bilayers on oil-infused surfaces. Proc. Natl. Acad. Sci. U.S.A. 2014, 111 (21), 7588-7593.

(44) Gersten, K.; Herwig, H. Strömungsmechanik. Grundlagen der Impuls-, Wärme-und Stoffübertragung aus Asymptotischer Sicht; Vieweg: Braunschweig, Wiesbaden, Germany, 1992.

(45) Incropera, F. P.; Dewitt, D. P. Fundamentals of Heat and Mass Transfer; John Wiley \& Sons: New York, 2002.

(46) Fedosov, A. I. Thermocapillary motion. Zh. Fiz. Khim. 1956, 30 (N2), 366-373.

(47) Korösi, G.; Kováts, E. S. Density and surface tension of 83 organic liquids. J. Chem. Eng. Data 1981, 26 (3), 323-332.

(48) Andrieu, C.; Beysens, D. A.; Nikolayev, V. S.; Pomeau, Y. Coalescence of sessile drops. J. Fluid Mech. 2002, 453, 427-438.

(49) Wong, T. S.; Kang, S. H.; Tang, S. K. Y.; Smythe, E. J.; Hatton, B. D.; Grinthal, A.; Aizenberg, J. Bioinspired self-repairing slippery surfaces with pressure-stable omniphobicity. Nature 2011, 477 (7365), 443-447. 